\title{
Mastalgia: Risk Factors and Treatment Strategies Used at Two Breast Clinics in Pakistan
}

\author{
Razia Bano ${ }^{1}$, Sohail Saqib Chatha ${ }^{2}$, Faiza Sana ${ }^{3}$, Zain Farooq ${ }^{4}$, Umar Farooq ${ }^{5}$, Abdullah Zakaullah ${ }^{6}$ \\ ${ }^{1}$ Consultant Breast Surgeon, Combined Military Hospital Rawalpindi, Punjab Pakistan \\ ${ }^{2}$ Consultant Surgeon, Combined Military Hospital Thall, Hangu, Khyber Pakhtunkhwa, Pakistan \\ ${ }^{3}$ Medical officer, Combined Military Hospital Rawalpindi, Punjab Pakistan \\ ${ }^{4}$ Resident surgeon, Combined Military Hospital Rawalpindi, Punjab Pakistan \\ ${ }^{5}$ Medical officer, Fauji Foundation Hospital, Rawalpindi, Punjab Pakistan \\ ${ }^{6}$ House officer, Fauji Foundation Hospital, Rawalpindi, Punjab Pakistan
}

\begin{abstract}
A BSTRACT
Background: Mastalgia is one of the commonest symptoms of breast disorder and a major cause of anxiety amongst women. The aim of this study was to determine the risk factors associated with the onset and severity of mastalgia, their association with various breast disorders, and response to strategies used for its treatment.

Material and Methods: This prospective descriptive study was carried out at Combined Military Hospitals (CMH) of Rawalpindi and Thall over a period of six months from January to July 2017. Women presenting to breast surgery clinic with complaints of breast pain, heaviness, and tenderness were included in the study. Patients were initially evaluated at the time of presentation, treatment was initiated, followed by re-evaluation after six months. A multiple logistic regression model was used to determine association of mastalgia with various conditions and their effect on severity of mastalgia.

Results: A total of 93 women presented to the breast clinics during this period. Cyclical mastalgia was noted in $39.8 \%$ women while $60.2 \%$ had non-cyclical mastalgia. In $41.9 \%$ patients no underlying pathology was noted, $49.5 \%$ patients had benign disorders while $8.6 \%$ had malignancy. Increased BMI $(P=.002)$ and lack of exercise $(P=.01)$ were associated with onset of mastalgia while use of oral contraceptive pills $(P<.001)$ was associated with low risk of mastalgia. Of all the patients, $45.1 \%$ responded to measures like reassurance, dietary and lifestyle modifications while $54.9 \%$ were treated with pharmacological therapy. Those diagnosed having underlying malignant disorder were treated according to the type and stage of malignancy.

Conclusions: Various factors affect the onset and severity of mastalgia and different treatment strategies can be used to alleviate the symptoms. Hence, complete and thorough evaluation of the patients presenting with mastalgia should be carried out to rule out any underlying pathology.

Key words: Onset of mastalgia, Oral contraceptive pills, Pharmacological treatment, Severity of mastalgia

Authors' Contribution:
${ }^{1}$ Conception; Literature research;
manuscript design and drafting; ${ }^{2,3}$ Critical
analysis and manuscript review; Dat
analysis; Manuscript Editing.

Received: June 22, 2020

Accepted: December 15, 2020
\end{abstract}

Cite this article. Bano R, Chatha SS, Sana F, Farooq Z, Farooq U, Zakaullah A. Mastalgia: Risk Factors and Treatment Strategies Used at Two Breast Clinics in Pakistan. J Islamabad Med Dental Coll. 2020; 9(4): 285-290. Doi: 10.35787/jimdc.v9i4.561

\author{
Funding Source: Nil \\ Correspondence: \\ Sohail Saqib Chatha \\ Email:drsohailchatha@hotmail.com \\ Article info: \\ Conflict of Interest: Nil
}




\section{Introduction}

Mastalgia, or breast pain, is considered as one of the commonest breast symptoms with which woman present in breast surgery clinics. It can affect up to $70 \%$ of women in their life time. ${ }^{1}$ Mastalgia is usually associated with various conditions such as infection, malignancy or extrathoracic conditions however fear of underlying malignancy is usually the cause for medical consultation. Mastalgia can be both noncyclical and cyclical. Its severity can range from mild to severe pain. The pain can be severe enough to the extent of interfering with routine life activities and can become a cause of anxiety and depression in certain cases. ${ }^{2}$ Exact pathogenesis of mastalgia remains to be undetermined however, certain factors like hormonal changes, age, smoking, stress, caffeine and duct ectasia have been associated with it. $^{3}$

In majority of woman presenting with mastalgia only reassurance is needed and it resolves the symptoms. In certain other conditions, various lifestyle modifications such as weight reduction, smoking cessation and exercise programmes can alleviate the symptoms. ${ }^{4,5}$ However certain subset of women with more intense pain need pain-relieving therapy in the form of oral and topical non-steroidal antiinflammatory drugs along with mechanical breast support and advice for consumption of low fat, high carbohydrate diet. Various hormonal agents such as danazol, bromocriptine and tamoxifen have also been used with certain success in the treatment of mastalgia. Due to variation in the prevalence and intensity of mastalgia and fear of its association with underlying breast cancer, complete investigation and treatment of mastalgia is essential. Treatment can range from simple assurance to dealing with the underlying cause. Despite being a common and benign issue mastalgia is the most common symptom for which breast consultation is done and is major cause of anxiety and concern amongst the affected women. There is no standardized treatment modality available and treatment and management is tailored on a case-to-case basis and is still a matter of debate and research in the literature. This study aimed to determine the risk factors associated with the onset and severity of mastalgia, their association with various breast disorders, and response to strategies used for its treatment.

\section{Material and Methods}

This prospective descriptive study was carried out at the Breast surgery clinics of $\mathrm{CMH}$ Rawalpindi, Punjab Pakistan and $\mathrm{CMH}$ Thall, Khyber Pakhtunkhwa Pakistan over a period of six months from January to July 2017. After obtaining ethical approval from Combined Military Hospital, Thall and written informed consent from the patients, a total of 93 woman fulfilling the inclusion criteria were enrolled in the study. Sample size was calculated using online sample size calculator with $95 \%$ confidence level, $10 \%$ margin of error and population size of approximately 1500 (number of women presenting to breast surgery clinics over the period of previous seven months). Women who presented with symptoms of breast pain, heaviness, burning sensation and tenderness were included, whereas women with already confirmed diagnosis of any benign or malignant breast disorder were excluded from the study. Detailed history and clinical examination were carried out. Intensity of mastalgia was determined with the help of Visual Analog Scale (VAS) ranging from 1 to 10 . Based on findings of history and examination, relevant investigations such as sonomammogram, mammogram, FNAC, Core biopsy etc. were done if needed in order to determine the association of mastalgia with any underlying pathology. Treatment was given on the basis of final diagnosis ranging from simple 
assurance to surgery. Patients were followed-up over a period of six months, were re-evaluated and resolution of symptoms noted.

All relevant information was collected and noted in a standardized manner, which included various demographic characteristics such as age, pre- or postmenopausal status, unilateral or bilateral symptoms and any associated symptoms such as nipple discharge etc., along with relevant family history. Results of the prescribed investigations along with final diagnosis and treatment prescribed were also noted. Patients were then followed over a period of six months and effectiveness of treatment was noted in terms of resolution of the mastalgia. Data was analyzed using SPSS version 20.0. Data was analyzed using descriptive statistics in the form of mean \pm standard deviation for quantitative variables and percentage for categorical variables. A multiple logistic regression model was used to determine association of mastalgia with various conditions and their effect on severity of mastalgia.

\section{Results}

A total of 93 females were evaluated for symptoms of mastalgia. Women ranged in age from $19-70$ years with mean age of $35 \pm 5.25$ years. Cyclical mastalgia was noted in $39.8 \%(n=37)$ women while $60.2 \%$ $(n=56)$ had non-cyclical mastalgia. Out of these $67.8 \%$ ( $n=63$ ) had mild to moderate mastalgia while $32.2 \%(n=30)$ had severe mastalgia. The mean score of mastalgia was $4.56 \pm 2.32$ according to VAS. Regarding menopausal status, mastalgia occurred in 76.3\% premenopausal women while $23.7 \%$ were postmenopausal. Most of the women presenting with mastalgia $(60.2 \% ; n=56)$ had complaints of unilateral mastalgia. In $41.9 \%$ ( $n=39$ ) patients, no underlying pathology was noted, while $49.5 \%(n=46)$ had benign disorders and only $8.6 \%(n=8)$ had underling malignancy which was confirmed with biopsy (Figure 1).

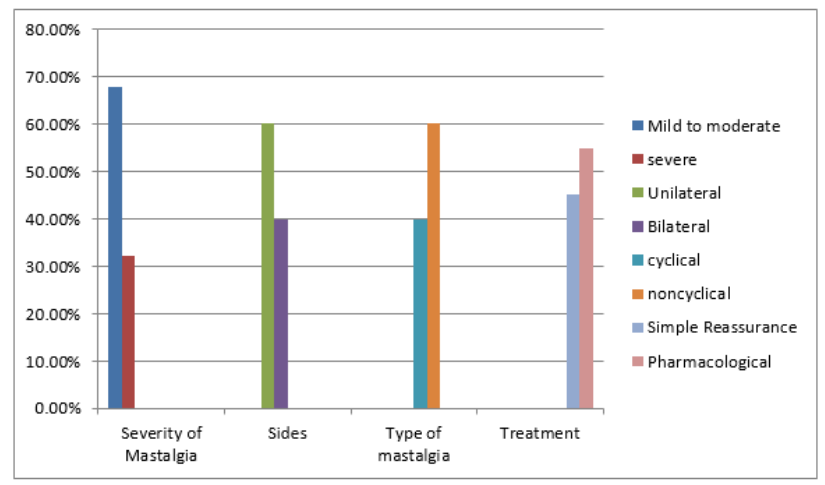

Figure 1: Bar chart showing comparison of frequency distribution of severity, laterality, types and treatment strategies of mastalgia.

Factors such as parity, marital status, educational status as well as duration and interval of menstrual cycle had no effect on mastalgia. Using multiple logistic regression model, age of marriage, increased BMI, lack of exercise were associated with onset of mastalgia while use of OCP was associated with low risk of mastalgia (Table I).

\begin{tabular}{|l|c|c|c|}
\hline \multicolumn{4}{|c|}{ Table I: Effect of various factors on onset of } \\
mastalgia
\end{tabular}

${ }^{*} P$-value $<.05$ was considered statistically significant

Regarding effect on severity of mastalgia anxiety, increased age and increased age at time of marriage were positively associated whereas regular exercise and OCP use had beneficial effect in reducing the severity of mastalgia (Table II). About $45.1 \%(n=42)$ patients responded to simple measures like reassurance, dietary and lifestyle modifications while $54.9 \%(n=51)$ were treated NSAIDS, Evening primrose oil (EPO), danazol and tamoxifen. Those having underlying malignancy diagnosed during 
workup of mastalgia were treated according to the underlying malignancy.

\begin{tabular}{|l|c|c|c|}
\hline \multicolumn{4}{|c|}{ Table II: Effect of various factors on severity of } \\
mastalgia
\end{tabular}

${ }^{*} P$-value $<.05$ was considered statistically significant

\section{Discussion}

In our study factors like age at marriage, increased $\mathrm{BMI}$ and lack of exercise had adverse effects on the onset of mastalgia, while use of OCP reduced the risk of onset of mastalgia. Factors like anxiety and increased age were associated with increased severity of mastalgia, while regular exercise and OCP use reduced the severity of mastalgia.

Mastalgia has been found to have association with various psychological disorders like anxiety and depression. ${ }^{6,7}$ In a study by Ramirez et al., increased stress level was associated with severity of mastalgia. ${ }^{8}$ Conflicting results were reported by Shobeiri et al. who found no association between depression/anxiety and mastalgia in women attending health centers of Hamadan City, $\operatorname{Iran}^{9}$ However various studies have suggested that clinicians dealing with patients suffering from mastalgia should inquire about anxiety, depression and any history of trauma. ${ }^{10,11}$ According to our study, $39.8 \%$ of woman had cyclical mastalgia which was closer to the results of Ader et al. who also reported $30 \%$ of woman suffering from cyclical mastalgia. ${ }^{12}$

Factors adversely associated with mastalgia in this study were age at marriage, increased BMI and lack of exercise, while use of OCP had beneficial effect on mastalgia. Few studies have shown association of mastalgia with smoking, caffeine consumption, ${ }^{6,13}$ pregnancy and trauma. In our study, lack of exercise was a determinant factor for onset of mastalgia. However, this is in contrast to a study by Brown et al. on female marathon runners, who reported that exercise can cause mastalgia. ${ }^{14}$ The severity of pain on the VAS was $4.56 \pm 2.32$ in our study, which is consistent with the findings of Deniz and co-workers with an average pain score of $4.54 \pm 2.1 .{ }^{15}$

About $41.9 \%$ of our patients had no underlying breast disorder, $49.5 \%$ had benign findings while only $8.6 \%$ had underlying malignancy. Similar results were found in another study carried out by Ader et al. ${ }^{16}$ where one third of the woman had normal mammographic findings, half had benign masses and only 3 cases had underlying malignancy. This indicates a poor association of mastalgia with underlying breast cancer, which can lead to anxiety and fear in woman suffering from mastalgia and can be a cause for needless surgical consultation.

Treatment of mastalgia is another matter of debate and ranges from simple reassurance to surgical therapies. In our study $45.1 \%$ of patients responded to simple measures like reassurance and lifestyle modifications. In the remaining cases, multiple modalities like NSAIDS, hormonal therapy, danazol and even surgery was used on case-to-case basis. In studies carried out by Ader et al. and AA Muhammad, ${ }^{17}$ it has been shown that after alleviating fear of breast cancer by proper evaluation, simple reassurance is a useful and firstline treatment option. In another study conducted in the Department of Breast Surgery, St George's Hospital London, authors argue that mastalgia may settle spontaneously without any treatment. ${ }^{18}$ Lifestyle modifications such as low fat, high carbohydrates diet, weight reduction and regular exercise along with use of well-fitting bras has also been found as effective means of treatment in a number of patients $^{19}$ and this fact was 
demonstrated in our study as well. In $54.9 \%$ of our patient's various pharmacological treatments including NSAIDS, Epo, danazol and various hormonal preparations like tamoxifen were used with good results. Other studies have verified these results. A study by Rosolowich et al. recommend that topical non-steroidal anti-inflammatory gel, such as diclofenac $2 \%$ is effective for pain control for localized treatment of mastalgia and that tamoxifen or danazol can be used as a treatment option, if other measures fail. ${ }^{20}$ In a study by Gupta et al. danazol was effective in alleviating mastalgia in $71 \%$ of patients, while tamoxifen was successful in $67 \%$ of cyclical mastalgia. ${ }^{21}$ Though few studies have shown some relief in breast discomfort with reduction mammoplasty, ${ }^{22,23}$ especially in patients with symptomatic macromastia, in general surgery it has no role in the management of mastalgia. This finding was corroborated in our study as well, where surgery was done only for the treatment of malignancy, and not for mastalgia.

This was an observational study with a small sample size. Studies with larger sample size are needed in order to validate the results of this study.

\section{Conclusion}

Non-cyclical, mild-to-moderate, unilateral mastalgia is more common in our study. Factors like age at marriage, increased BMI and lack of exercise are associated with high-risk of onset of mastalgia. Anxiety, increased age, and increased age at time of marriage are positively associated whereas regular exercise and OCP use reduced the severity of mastalgia. Different treatment options are available, ranging from simple assurance to pharmacological treatments and rarely surgery depending upon any associated disorder.

\section{References}

1. Eren T, Aslan A, Ozemir IA, Baysal H, Sagiroglu J, Ekinci $O$, et al. Factors effecting mastalgia. Breast Care. 2016; 11(3): 188-93. Doi: 10.1159/000444359.

2. Smith RL, Pruthi S, Fitzpatrick LA. Evaluation and management of breast pain. Mayo Clin Proc 2004; 79(3): 353-72. Doi: 10.4065/79.3.353.

3. Norlock FE. Benign breast pain in women: a practical approach to evaluation and treatment. J Am Med Women Assoc. 2002; 57(2): 85-90. PMID: 11991427.

4. Joyce DP, Alamiri J, Lowery AJ, Downey E, Ahmed A, McLaughlin $\mathrm{R}$, et al. Breast clinic referrals: can mastalgia be managed in primary care? Ir J Med Sci. 2014; 183(4): 639-42. Doi: 10.1007/s11845-0131066-z.

5. Shobeiri $F$, Jenabi $E$. The effects of vitamin $E$ on muscular pain reduction in students affected by premenstrual syndrome. Iran J Obstet Gynecol Infertil. 2014; 17(96): 1-5.

6. Johnson KM, Bradley KA, Bush K, Gardella C, Dobie DJ, Laya MB. Frequency of mastalgia among women veterans. Association with psychiatric conditions and unexplained pain syndromes. J Gen Intern Med. 2006; 21(3): S70-5. Doi: 10.1111/j.15251497.2006.00378.x

7. Colegrave S, Holcombe C, Salmon P. Psychological characteristics of women presenting with breast pain. J Psychosom Res. 2001; 50: 303e7. Doi: 10.1016/s0022-3999(01)00196-9.

8. Ramirez AJ, Jarret SR, Hamed H, Smith P, Fentiman IS. Psychosocial adjustment of women with mastalgia. Breast J. 1995; 4(1): 48-51.

9. Shobeiri F, Oshvandi K, Nazari M. Cyclical mastalgia: Prevalence and associated determinants in Hamadan City, Iran. Asian Pac J Trop Biomed. 2016; 6(3): 2758. Doi: 10.1016/j.apjtb.2015.12.008.

10. Shobeiri F, Oshvandi K, Nazari M. Clinical effectiveness of vitamin $E$ and vitamin $B 6$ for improving pain severity in cyclic mastalgia. Iran J Nurs Midwifery Res. 2015; 20(6): 723-7. Doi: 10.4103/1735-9066.170003.

11. Plu-Bureau G, L^e MG, Sitruk-Ware R, Thalabard JC. Cyclical mastalgia and breast cancer risk: results of a French cohort study. Cancer Epidemiol Biomarkers Prev. 2006; 15(6): 1229-31. Doi:10.1158/1055-9965.

12. Ader DN, South-Paul J, Adera T, Deuster PA. Cyclical mastalgia: prevalence and associated health and behavioral factors. J Psychosom Obstet Gynaecol. 2001; 22(2): 71-6. Doi:10.3109/01674820109049956

13. Kataria K, Dhar A, Srivastava A, Kumar S, Goyal A. A systematic review of current understanding and management of mastalgia. Indian J Surg. 2014; 76(3): 217-22. Doi: 10.1007/s12262-013-0813-8. 
14. Brown N, White J, Brasher A, Scurr J. The experience of breast pain (mastalgia) in female runners of the 2012 London Marathon and its effect on exercise behaviour. Br J Sports Med. 2014; 48(4): 320-5. Doi: 10.1136/bjsports-2013-092175.

15. Deniz Koçoğlu, Serife Kurşun, Belgin Akin, Kamile Altuntug. Mastalgia and associated factors: a crosssectional study. Agri. 2017; 29(3): 100-8. Doi: 10.5505/agri.2017.91069.

16. Ader DN, Shriver CD, Browne MW. Cyclical mastalgia: premenstrual syndrome or recurrent pain disorder? J Psychosom Obstet Gynaecol. 1999; 20(4): 198-202. Doi: 10.3109/01674829909075596.

17. Mohammed A. Predictive factors affecting axillary lymph node involvement in patients with breast cancer in Duhok: Cross-sectional study. Ann Med Surg. 2019; 44: 87-90. Doi: 10.1016/j.amsu.2019.07.011.

18. Gumm R, Cunnick GH, Mockbel K. Evidence for management of mastalgia. Curr Med Res Optn. 2004; 20(5): 681-4. Doi: 10.1185/030079904125003377.
19. Olawalye A, Withiam-Leitch M, Danakas G, Kahn K. Mastalgia: a review of management. J Reprod Med. 2005; 50(12): 933-9. PMID: 16444894.

20. Rosolowich V, Saettler E, Szuck B, Lea RH, Levesque $\mathrm{P}$, Weisberg F et al. Mastalgia. J Obstet Gynaecol Can. 2006; 28(1): 49-71. Doi: 10.1016/S17012163(16)32027-8.

21. Gupta P, Verma V, Gupta R, Kumar A, Singh SP, Gupta UK, et al. Role of tamoxifen and danazol in mastalgia: prospective controlled trial. ISJ. 2016; 3(2): 553-6. Doi: 10.18203/2349-2902.isj20160703.

22. Chadbourne EB, Zhang S, Gordon MJ, Ro EY, Ross SD, Schneider PR, et al. Clinical outcomes in reduction mammoplasty: a systematic review and metaanalysis of published studies. Mayo Clin Proc. 2001; 76(5): 503-10. Doi: 10.4065/76.5.503.

23. Blomqvist L, Eriksson A, Brandberg Y. Reduction mammoplasty provides long-term improvement in health status and quality of life. Plast Reconstr Surg. 2000; 106(5): 991-7. Doi: 10.1097/00006534200010000-00005. 